Tôhoku Math. Journ.

39 (1987), 101-103.

\title{
THE MORDELL-WEIL RANK OF ELLIPTIC CURVES
}

\author{
JASBIR Singh ChAHAL
}

(Received February 18, 1986)

Let $E$ be an elliptic curve defined over a number field $k$ (of finite degree). The Mordell-Weil Theorem states that the group $E(k)$ of $k$ rational points of $E$ is finitely generated. Consequently, the group $E(k)_{\text {tor }}$ of points of finite order of $E(k)$ is finite.

Let $L$ be the field obtained by adjoining to $k$ all the roots of unity. It has been shown by Ribet (cf. [1]) that even for the infinite extension $L / k$, the group $E(L)_{\text {tor }}$ is still finite. However, as we will show here, $E(L)$ can never be finitely generated.

We will denote the Mordell-Weil rank of $E$ over $k$ by $r_{k}(E)$. It is the maximum number of free generators of $E(k)$.

THEOREM. Suppose $E$ is an elliptic curve defined over $\boldsymbol{Q}$ and $r_{0}$ is a positive integer. Then there is a finite extension ${ }^{* 1} K$ of $\boldsymbol{Q}$, such that $r_{K}(E)>r_{0}$. Moreover, there is a constant $c=c(E)$, such that the degree of extension $[K: \boldsymbol{Q}] \leqq c 2^{r_{0}}$.

Proof. Suppose $E$ is given in the Weierstrass form

$$
y^{2}=x^{3}+A x+B \quad(A, B \in \boldsymbol{Q}) .
$$

The polynomial $f(x)=x^{3}+A x+B$ has distinct roots $e_{1}, e_{2}, e_{3}$ and factors as

$$
f(x)=\left(x-e_{1}\right)\left(x-e_{2}\right)\left(x-e_{3}\right)
$$

in its splitting field $k$. We may consider $E$ to be defined over $k$. Let $P_{j}=\left(x_{j}, y_{j}\right), j=1, \cdots, m$, be all the points of finite order of $E(L)-\{O\}$.

For any $P=(x, y)$ in $E(k)$, the factors $x-e_{1}, x-e_{2}, x-e_{3}$ of $y^{2}$ in (2) are almost relatively prime. More precisely, there are finitely many $d_{1}, \cdots, d_{n}$ in $k$, such that for any $P=(x, y)$ in $E(k)$, each factor is of the form

$$
x-e_{i}=d_{j} z^{2}
$$

for some $j(1 \leqq j \leqq n)$ and $z$ in $k$. To prove this let $S$ denote any finite set of primes (including all the Archemedian ones) of $k$. By Dirichelet's

\footnotetext{
* See the remark at the end of the paper.
} 
theorem, the group $U_{k}(S)$ of $S$-units of $k$, i.e.,

$$
U_{k}(S)=\left\{x \in k \mid \operatorname{ord}_{\mathfrak{p}}(x)=0, \mathfrak{p} \notin S\right\}
$$

is finitely generated, say by $\eta_{1}, \cdots, \eta_{r}$. For what follows, we may suppose that all $e_{i} \in \mathcal{O}_{k}$, the ring of integers of $k$. Choose a finite $S$ containing all the prime divisors of

$$
\prod_{i<j}\left(e_{i}-e_{j}\right)
$$

and with the property that

$$
\mathcal{O}_{k}(S)=\left\{x \in k \mid \operatorname{ord}_{\mathfrak{p}}(x) \geqq 0, \mathfrak{p} \notin S\right\}
$$

is a principal ideal domain.

Now let $P=(x, y)$ be a $k$-rational point on $E$. A prime divisor $\mathfrak{p}$ of $x-e_{1}$ and $x-e_{2}$ must divide $e_{1}-e_{2}$. Thus for any $\mathfrak{p} \notin S$, the exponent $\operatorname{ord}_{\mathfrak{p}}\left(\left(x-e_{2}\right)\left(x-e_{1}\right)^{-1}\right)$ in the factorization of $\left(x-e_{2}\right)\left(x-e_{1}\right)^{-1}$ is even, say $2 a_{\mathfrak{p}}$. If

$$
z_{1}=\prod_{\mathfrak{p} \notin S} \pi_{\mathfrak{p}}^{-a_{\mathfrak{p}}},
$$

where $\pi_{\mathfrak{p}}$ is a uniformizing parameter at $\mathfrak{p}$, then it is clear that $\left(x-e_{2}\right)\left(x-e_{1}\right)^{-1} z_{1}^{2}$ is an $S$-unit. So for some $m_{i} \in \boldsymbol{Z}$

$$
x-e_{2}=\left(x-e_{1}\right) \eta_{1}^{m_{1}} \cdots \eta_{r}^{m_{r}} z_{1}^{-2} .
$$

Similarly

$$
x-e_{3}=\left(x-e_{1}\right) \eta_{1}^{n_{1}} \cdots \eta_{r}^{n_{r}} z_{2}^{-2} .
$$

Substituting (4) and (5) in (2), we get

$$
x-e_{1}=\eta_{1}^{\alpha_{1}} \cdots \eta_{r}^{\alpha_{r}} z^{2} \quad\left(0 \leqq \alpha_{i} \leqq 1\right) .
$$

We may suppose that no $d_{i} d_{j}^{-1}(i \neq j)$ is a square in $k$. Now choose $t$ in $k$, such that

(A) $d_{i} d_{j}^{-1} t$ is not a square in $k$ for any pair $i, j$ (including $i=j$ ) and

(B) $x_{0}=e_{1}+d_{1} t \in \boldsymbol{Q}$ and is not a root of the polynomial $g_{j}(x)=f(x)-y_{j}^{2}$ for all $j=1, \cdots, m$.

If we put $y_{0}=(f(x))^{1 / 2}$ with $x_{0}$ as in (B), then $y_{0}$ is not in $k$, because otherwise (B) and (3) would contradict (A). However, for a root $\zeta$ of unity, we have $y_{0} \in \boldsymbol{Q}(\zeta)$. Therefore, the point $P_{0}=\left(x_{0}, y_{0}\right)$ is in $E(L)$. By (B), $P_{0}$ is not a point of finite order. If we put $K_{1}=k\left(y_{0}\right)$, then $r_{K_{1}}(E)>r_{k}(E)$ and $\left[K_{1}: k\right]=2$. We repeat the process with $k$ replaced by $K_{1}$ to get a quadratic extension $K_{2} \subseteq L$ of $K_{1}$, such that $r_{K_{2}}(E)>r_{K_{1}}(E)$. This process now may be continued until $r_{K_{i}}(E)$ exceeds $r_{0}$. To prove the last assertion, we take $c=[k: Q]$. 
Corollary. For no elliptic curve $E$ defined over $\boldsymbol{Q}$, is $E(L)$ finitely generated.

REMARK. The finite extension $K$ is actually the composite of the splitting field $k$ of $x^{3}+A x+B$ and $L^{\prime}$, where $L^{\prime}$ is a composite of quadratic fields with galois group $\operatorname{Gal}\left(L^{\prime} / \boldsymbol{Q}\right) \cong(\boldsymbol{Z} / 2 \boldsymbol{Z})^{r_{0}}$. Moreover, if the discriminant

$$
\Delta=-\left(4 A^{3}+27 B^{2}\right)
$$

of $E$ is a square in $\boldsymbol{Q}$, then $k$ and hence $K$ is abelian.

Acknowledgement. The author would like to thank Professor Wolfgang M. Schmidt for inviting him to Boulder (April 15-May 15, 1985), where this work was done.

\section{REFERENCE}

[1] K. A. RIBET, Torsion points of abelian varieties in cyclotomic extensions (Appendix to N. M. Katz and S. Lang, Finiteness theorems in geometric class field theory), Enseign. Math. (2) 27 (1981), 315-319.

Department of Mathematics

BRIGHAM YOUNG UNIVERSITY

Provo, UT 84602

U.S.A. 
